\title{
Cell sizing in chronic lymphoproliferative disorders: an aid to differential diagnosis
}

\author{
H D Alexander, G M Markey, R L Nolan, T C M Morris
}

\begin{abstract}
Aims: To determine if leucocyte volume distribution analysis (LVDA), obtained using a Coulter Counter Model S Plus IV, can be used to aid differentiation of chronic lymphoproliferative disorder (CLPD) subtypes.
\end{abstract} lymphocyte distribution width were measured on each patient $(n=90)$ using gram. The mean lymphocyte volume was taken as the mean of the values on either side of the peak at half maximum height. The lymphocyte distribution width was taken as the range of cell values between the two values used to calibrate the mean lymphocyte volume. A template showing typical histograms from commonly occurring CLPD was also produced on an acetate sheet. This was used to examine the histogram from each new patient to evaluate its usefulness as an alternative to the calculation of mean lymphocyte volume and lymphocyte distribution width.

Results: Mean lymphocyte volume and lymphocyte distribution width were significantly higher in $B$ cell lymphocytic leukaemia of mixed cell type (B CLL/PL), $B$ cell non-Hodgkin's lymphoma with peripheral blood spill, hairy cell leukaemia and $T$ cell prolymphocytic leukaemia than in B cell chronic lymphocytic leukaemia (B CLL). The mean lymphocyte volume, but not the lymphocyte distribution width, was also significantly higher in $T$ cell chronic lymphocytic leukaemia than in B CLL. The template gave an immediate preliminary indication of possible subtype(s) of disorder and could be used as an alternative to measurement of mean lymphocyte volume and lymphocyte distribution width.

Conclusions: Electronic haematology analysers producing an LVDA provide a useful, cost effective cell sizing analysis which can aid the differentiation of subtypes of CLPD.

Haematology, Belfast City Hospital, Belfast BT9 7AD, Northern Ireland

H D Alexander

G M Markey

R L Nolan

T C M Morris

Correspondence to: Dr $\mathrm{H}$ Denis Alexander

Accepted for publication 20 March 1992

$(\mathcal{F}$ Clin Pathol 1992;45:875-879)

The primary basis for differential diagnosis of chronic lymphoid leukaemias has traditionally been the morphology of the cells in peripheral blood and bone marrow smears. The availability of a wide spectrum of cytochemical and immunological markers, supplemented by electron microscopy, cytogenetic studies, and
Methods: Mean lymphocyte volume and a hard copy of an amplified LVDA histo-

histological examination of biopsied lymph nodes and spleen, has helped elucidate the heterogenous nature of these leukaemias. The crucial role of immunophenotyping and electron microscopy is recognised in the recent French American British (FAB) proposals for the classification of the chronic lymphoid leukaemias, ${ }^{1}$ but these techniques may not all be available in some centres.

The certain morphological identification of subtypes in the FAB classification can be difficult, especially if peripheral blood or bone marrow smears are not well spread and the leukaemic cells are present in small numbers. The identification of small numbers of prolymphocytes in the peripheral blood of a patient with $\mathrm{T}$ helper prolymphocytic leukaemia (T-PLL), who had been successfully treated with deoxycoformycin ${ }^{2}$ and who had begun to relapse, was in our experience difficult, even with the availability of a wide spectrum of cell markers.

Cell volume studies have been used by various workers to study leukaemic cells. ${ }^{310}$ These studies have frequently utilised the operative impedence instruments marketed by Coulter Electronics Limited. More recently, Robinson et al carried out a morphometric analysis of B cell leukaemias, ${ }^{11}$ where they studied cell size, nuclear:cytoplasmic ratio, chromatin condensation, size of nucleolus and degree of irregularity of nuclear and cytoplasmic outlines, and Ozari et al ${ }^{12}$ used large unstained cell counts on a Technicon H6000 analyser to differentiate B CLL from other B cell leukaemias.

The present generation of Coulter Counter instruments includes models which provide an LVDA in the form of a three part differential white cell count, and a white cell volume distribution curve may be printed for each sample analysed. We assessed the usefulness of this method of cell sizing, using a Coulter Counter Model S Plus IV, in the diagnosis and monitoring of treatment of patients with a range of CLPD.

\section{Methods} lenediamine tetra-acetic acid $\left(\mathrm{K}_{2}\right.$ EDTA) were obtained from patients with the following group of disorders: B CLL, B CLL/PL, B prolymphocytic leukaemia (B PLL), leukaemic phase of B cell non-Hodgkin's lymphoma (B NHL), hairy cell leukaemia (HCL), splenic lymphoma with circulating villous lymphocytes (SLVL), T prolymphocytic leukaemia 


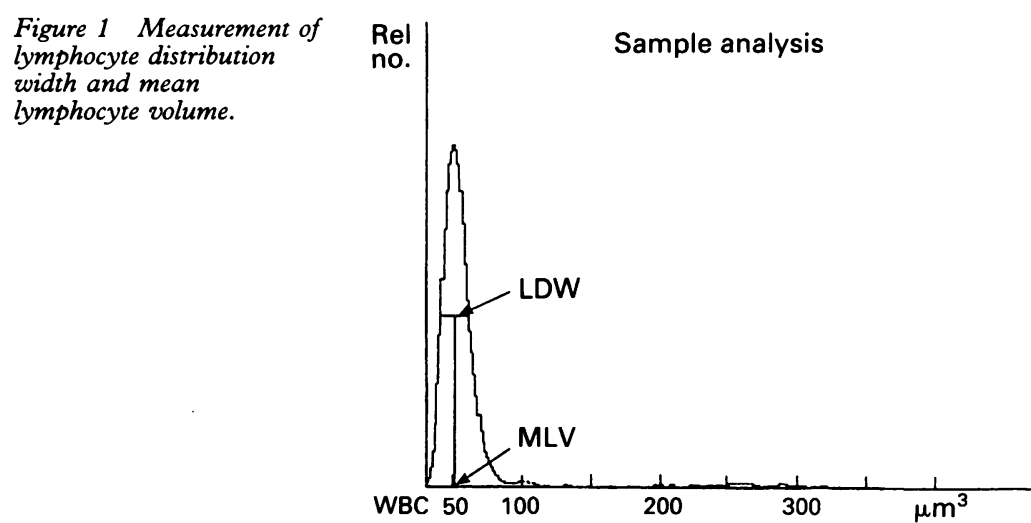

$(\mathrm{CD} 4+)$ (T PLL), large granular lymphocytosis $(\mathrm{CD} 8+)$ and Sézary syndrome. In each case the diagnosis was based on clinical, morphological, cytochemical and immunological data and confirmed where appropriate by electron microscopy, and the classifications used were those recommended by the FAB group. ${ }^{1}$

A Coulter Counter Model S Plus IV was used for cell sizing and a hard copy of an amplified LVDA histogram was produced on each patient. This is achieved in the Coulter Counter Model S Plus IV by lysis of red blood cells and partial lysis of white cells by a quaternary ammonium base. This results in the collapse of white cell membranes around the nucleus and thus the cell volumes are not those of intact cells, but represent the nucleus, cell membrane collapsed around the nucleus, and varying numbers of intracytoplasmic granules. The populations lying between $35-90 \mathrm{fl}$, $90-160 \mathrm{fl}$ and $160-450 \mathrm{fl}$ are representative of lymphocytes, mononuclear cells, and granulocytes, respectively.

Full blood counts were carried out between 30 minutes and two hours after withdrawal of the blood, which allowed the sample to stabilise in the anticoagulant, but analysis to be completed before clinically important leucocyte degeneration occurred. Because of the

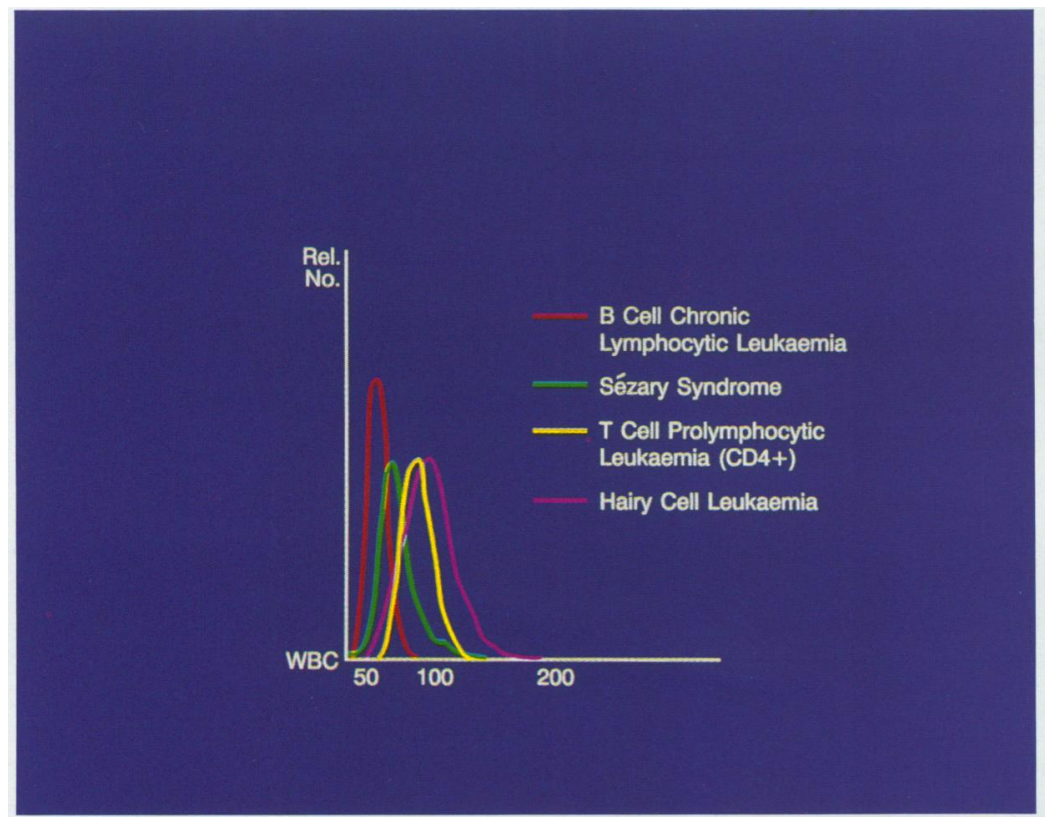

Figure 2 Lymphocyte volume distribution analysis template. very wide white cell count range in the patients studied (counts up to $735 \times 10^{9} / 1$ ), blood was diluted in Isoton III where necessary. This produced white cell counts in the range 10-20 $\times 10^{9} / 1$ for analysis on each patient, which avoided possible right-shifted histograms due to coincidence counts in patients with high white cell counts. Blood samples from patients with white cell counts of less than $20 \times 10^{9} / 1$ were processed without prior dilution.

Mean lymphocyte volumes (MLV) were measured using the method described by Bessman and Johnson, ${ }^{13}$ based on a Gaussian distribution of red blood cells. The cell volume at half maximum on either side of the peak was determined as the precise peak was difficult to locate due to rounding. The mean lymphocyte volume was then taken as the mean of these two values (fig 1 ).

A measurement of dispersion was also made. This we called the lymphocyte distribution width (LDW), and it was taken as the range of cell volumes between the two values used to calculate the mean lymphocyte volume (fig 1).

The pattern of each histogram was examined for evidence of twin peaks or a "shoulder" on either upward or downward slopes, which might represent a subpopulation of lymphoid cells. Finally, characteristic lymphoid cell histograms for a variety of CLPD were drawn on an A4 acetate sheet (fig 2). The acetate sheet was used as a template and placed over each LVDA histogram. The axes were aligned and the patient's lymphoid cell volume distribution examined against those on the acetate sheet.

Statistical analysis of results was carried out by the Mann Whitney U test, using Minitab Statistical Software.

\section{Results}

Typical individual results for a range of CLPD are shown in fig 3 and combined results are summarised in the table. The B CLL group showed the lowest mean lymphocyte volume and hairy cell leukaemia the highest. B CLL PL and B NHL occupied intermediate positions with B NHL showing a wider range of mean lymphocyte volume values. The other B cell lymphoproliferative disorders studied (SLVL, B PLL) had mean lymphocyte volumes clearly different from B CLL, although only one patient was analysed in each of these categories. The highest mean lymphocyte volumes in $\mathrm{T}$ cell disorders were found in T PLL and the lowest in T CLL, with the one patient with Sézary syndrome studied occupying an intermediate position.

Statistical analysis showed significant differences at the $1 \%$ level between mean lymphocyte volume in B CLL and B CLL/PL ( $p<0.01 ; 95 \%$ confidence limits for difference in medians -14.5 to -7.0 ), B CLL and B NHL ( $p<0.01 ; 95 \%$ confidence limits -12.5 to -6.0 ), and B CLL and T PLL ( $p<0.01 ; 95 \%$ confidence limits -32.5 to $-16 \cdot 0)$. Significant differences at the $5 \%$ level were found when mean lymphocyte volume results in B CLL were compared with those in 
Figure 3 Lymphocyte volume distribution analysis for a range of lymphoproliferative disorders.
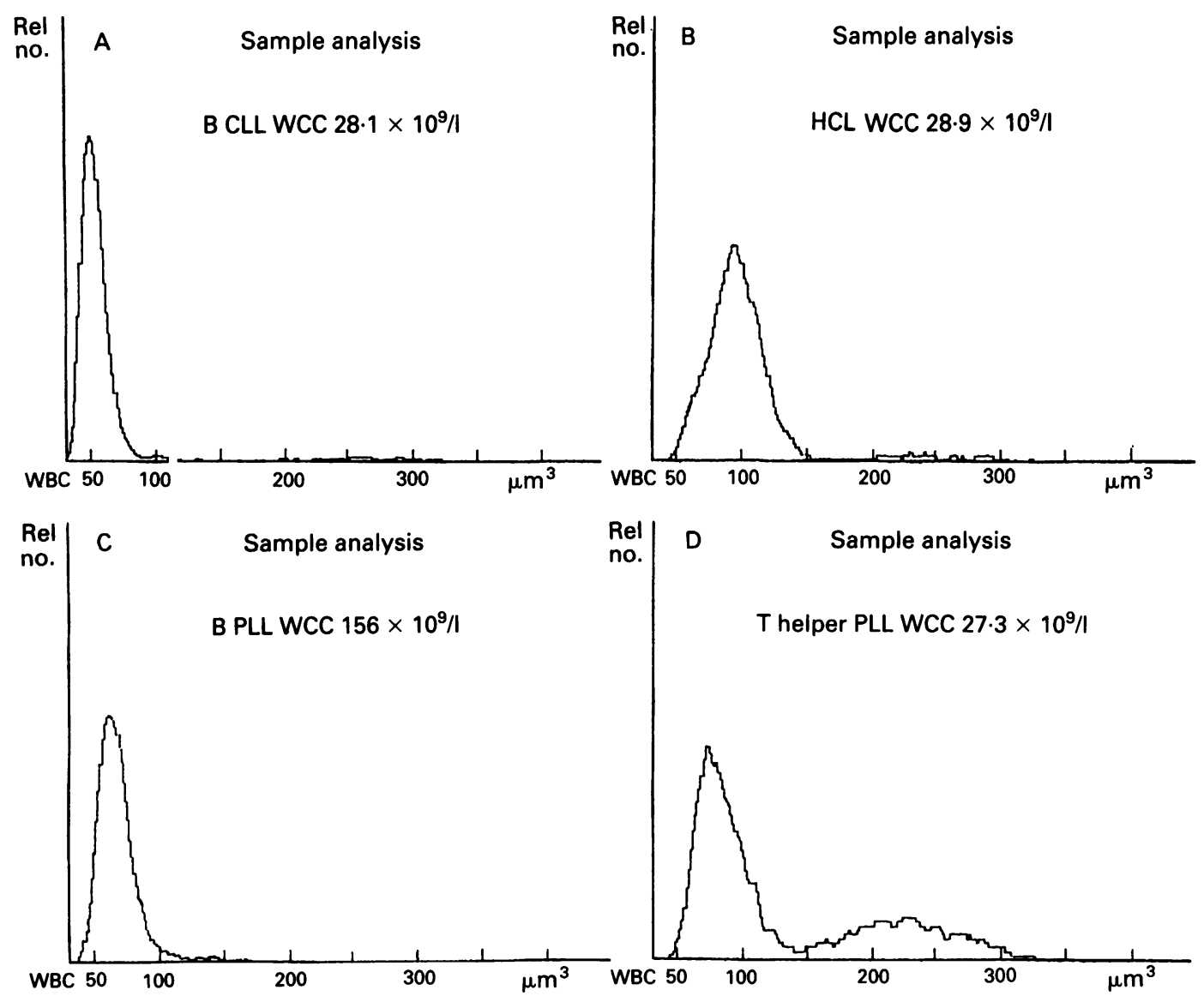

\begin{tabular}{l|l} 
Rel & Do. \\
&
\end{tabular}

Sample analysis

T heiper PLL WCC $27.3 \times 10^{9} / 1$
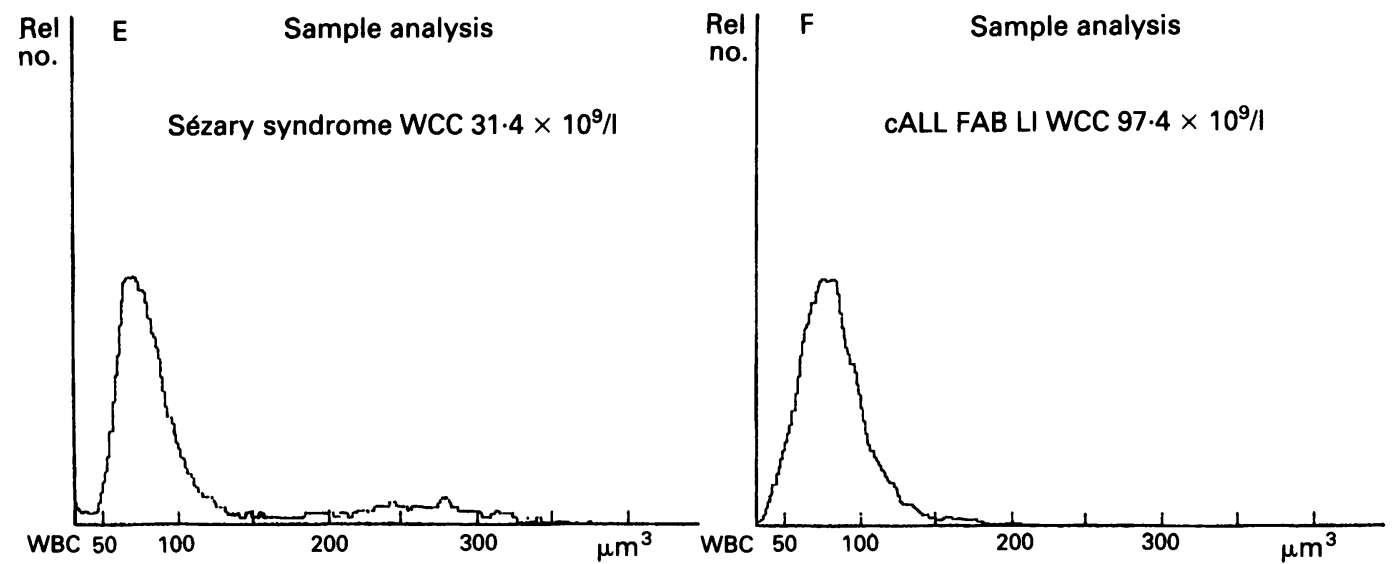

hairy cell leukaemia ( $p=0.0165 ; 95 \%$ confidence limits -41.6 to -29.5$)$ and $T$ CLL $(\mathrm{p}=0.0235 ; 95 \%$ confidence limits -9.0 to $-1 \cdot 3)$.

Median lymphocyte distribution width values in B cell disorders ranged from B CLL at the lower end to hairy cell leukaemia at the upper, with B CLL/PL and B NHL once again

Lymphocyte cell sizing in chronic lymphoproliferative disorders

\begin{tabular}{lll}
\hline Disorder & $\begin{array}{l}\text { Median mean lymphocyte } \\
\text { volume }(f) \\
\text { (interquartile range) }\end{array}$ & $\begin{array}{l}\text { Median lymphocyte } \\
\text { distribution width }(f) \\
\text { (interquartile range) }\end{array}$ \\
\hline B CLL $(\mathrm{n}=51)$ & $54 \cdot 0(50 \cdot 0,55 \cdot 0)$ & $20 \cdot 8(19 \cdot 8,22 \cdot 9)$ \\
B CLLPL $(\mathrm{n}=8)$ & $63 \cdot 5(60 \cdot 3,66 \cdot 6)$ & $27 \cdot 9(25 \cdot 0,35 \cdot 4)$ \\
B PLL $(\mathrm{n}=1)$ & $70 \cdot 8$ & $33 \cdot 3$ \\
B NHL $(\mathrm{n}=19)$ & $62 \cdot 5(56 \cdot 0,66 \cdot 5)$ & $25 \cdot 0(22 \cdot 9,29 \cdot 2)$ \\
SLVL $(\mathrm{n}=1)$ & $72 \cdot 9$ & $37 \cdot 5$ \\
Hairy cell leukaemia $(\mathrm{n}=2)$ & $89 \cdot 6$ & $45 \cdot 9$ \\
T PLL $(\mathrm{n}=4)$ & $77 \cdot 6$ & $27 \cdot 6$ \\
T CLL $(\mathrm{n}=3)$ & $58 \cdot 0$ & $18 \cdot 7$ \\
Sézary syndrome $(\mathrm{n}=1)$ & $63 \cdot 6$ & $22 \cdot 9$ \\
\hline
\end{tabular}

occupying intermediate positions. SLVL and B PLL had higher lymphocyte distribution width values than the median B CLL figure, but only one patient was analysed in each category. In the $\mathrm{T}$ cell lymphoproliferative disorders median lymphocyte distribution width results were higher in T PLL than in T CLL with Sézary syndrome occupying an intermediate position.

Statistical analysis showed significant differences at the $1 \%$ level between lymphocyte distribution width in B CLL and B CLL/PL ( $p<0.01 ; 95 \%$ confidence limits -10.4 to $-3.8)$, B CLL and B NHL ( $<<0.01 ; 95 \%$ confidence limits $-6 \cdot 2$ to $-2 \cdot 1)$, and B CLL and T PLL ( $<<0.01 ; 95 \%$ confidence limits -12.5 to $-3 \cdot 1)$. A significant difference at the $5 \%$ level was found between lymphocyte distribution width in B CLL and hairy cell leukaemia ( $p=0.0163 ; 95 \%$ confidence limits $-31 \cdot 2$ to $-16 \cdot 7)$. No significant difference 
Figure 4 Lymphocyte volume distribution analysis on a patient with T-PLL $(C D 4+)(A)$ in remission and $(B-F)$ in relapse.
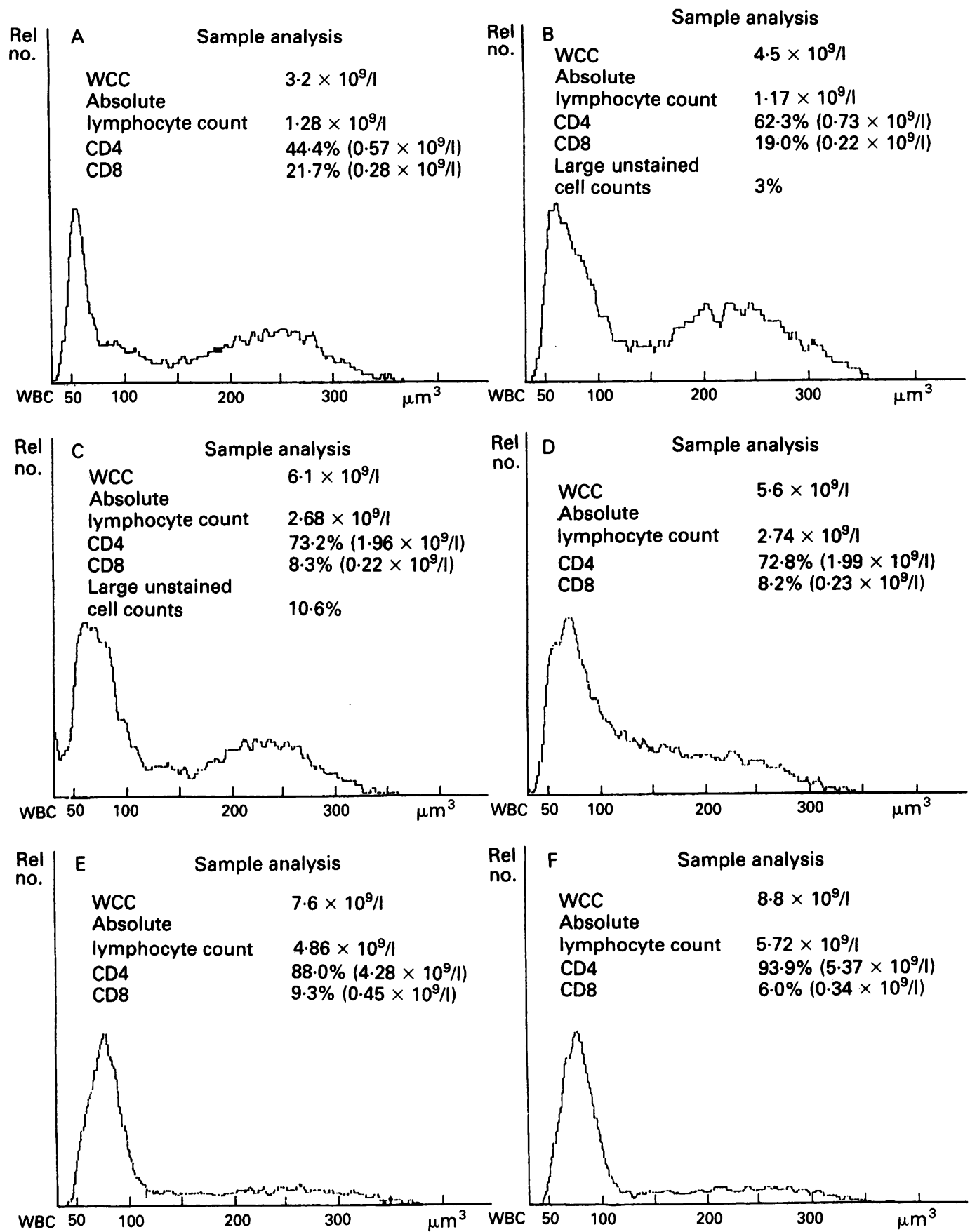

was found between lymphocyte distribution width in B CLL and T CLL $(p=0.3362)$.

In practice, the use of the A4 template proved a quick and satisfactory method for assessment of white cell histograms. Comparison of individual histograms (fig 3) with the template (fig 2) gave an immediate indication of the most probable lymphoproliferative disease subgroup involved. T PLL(D), hairy cell leukaemia (B), Sézary syndrome (E) were all clearly distinguishable from B CLL, while B PLL(C) gave a less pronounced but still obvious right-shifted plot. A case of common acute lymphoblastic leukaemia (cALL) (FAB L1) is included in fig 3 for comparison.

Figure 4 illustrates the leucocyte volume histograms of a patient with T PLL $(\mathrm{CD} 4+)$ (A) in remission after treatment with deoxycoformycin and (B-F) during subsequent relapse. The histogram in fig $4 \mathrm{~B}$ is obviously abnormal with a distinct shoulder on the downward slope, yet at this stage prolymphocytes were not readily identifiable in the peripheral blood smear. At this stage the absolute $\mathrm{CD} 4$ + lympocyte count was well within the reference range used in this laboratory $\left(0.47-1.93 \times 10^{9} / 1\right)$ and the large unstained cell count on a Technicon Hemalog $\mathrm{D} / 90$ was also within the normal range at $3 \%$. Subsequent monitoring of the patient is illustrated in fig $4 \mathrm{C}-\mathrm{F}$ : the absolute $\mathrm{CD} 4+$ increased large unstained cell count developed (fig 4C), and the lymphoid cell histogram moved further to the right.

\section{Discussion}

This study has shown that useful data are available for every patient with CLPD who has lymphocyte count continued to rise, an 
a routine full blood count carried out at diagnosis, using a Coulter Counter Model S Plus IV or similar instrument. The cell size distribution of the malignant lymphoid cells can be assessed by examining a hard copy of the LVDA histogram, most easily by using an acetate template containing typical white cell sizing histograms of various lymphoproliferative disorders. In the patients described this simple procedure allowed all other lymphoproliferative disorders studied to be differentiated from B CLL. This aspect would be particularly important in those laboratories investigating small numbers of these disorders or where immunophenotyping is not performed routinely, allowing samples which might otherwise have been accepted as typical B CLL to be referred to other centres for further investigation.

The wide spectrum of known CLPDs ${ }^{1}$ and their varying responses to different forms of treatment make it imperative that correct classification is achieved. The cell sizing procedure described here is a simple and useful extension of existing technology, alerting to the presence of lymphoproliferative disease other than B CLL and indicating the necessity for further investigations. Whether the procedure can differentiate between individual non-B CLL disorders cannot be decided from the present study due to the small number of patients in some of the groups but the data so far suggest that this may be possible. If so, this additional parameter may prove a valuable tool in the differential diagnosis of these disorders.

Of further practical value is the ability to detect small numbers of leukaemic cells in the peripheral blood of a patient relapsing after remission. As shown in fig 4, significant changes in the lymphocyte population of the patient with T PLL were occurring even though his white cells and absolute CD4 + and CD8 + numbers were within the normal range, as was his Hemalog D/90 large unstained cell count. Comparison of data obtained at intervals from patients with lymphoproliferative disorders may be a simple and valuable indicator not only of recurrence of disease as shown, but of progression of B CLL through B CLL/PL, to B PLL.

A limitation of the method is the fact that the cell membrane is collapsed on to the nucleus before sizing, and thus results may be more representative of nuclear size than overall cell size. Robinson et al, ${ }^{11}$ whose study was confined to B cell lymphoproliferative disorders, measured the cross-sectional area of the cells after separation over Lymphoprep and fixation for electron microscopy. An IBAS 2 computed image analysis system was used for the morphometric analysis. Nevertheless, in the three disorders common to both studies (B CLL, B PLL, and hairy cell leukaemia) they found the same hierarchy of cell size, with hairy cell leukaemia cells being the largest followed by $B$ PLL cells and B CLL cells. Thus while neither method is a true measure of overall cell volume, there is good correlation between these studies in those disorders common to both. Furthermore, using a Coulter Counter Model ZB1 linked to a Coulter Channeliser, Melo et $a l,{ }^{10}$ in a study of B CLL, B PLL, SLVL, hairy cell leukaemia and hairy cell leukaemia varient (HCLV) again found the same hierarchy of cell size, with HCLV, not included in the present study, occupying a position between SLVL and hairy cell leukaemia. However, unlike these other two studies, the method presented here does not require any specialised equipment.

The mean cell volume is one of the most reproducible full blood count parameters and cell sizing of leukaemic lymphoid cells seems to be similarly reproducible. The results described here were produced on a Coulter Counter Model S Plus IV, but other electronic cell counters producing a leucocyte volume distribution analysis should be similarly suitable for the procedure. While measurement of the mean lymphocyte volume and lymphocyte distribution width can be circumvented by use of a template as described in this study, the data to produce these parameters are accessible in the electronic cell counter, and instrument manufacturers may consider it appropriate that the lymphocyte equivalent of the mean cell volume and red cell distribution width be made available in future instruments.

We thank Maureen Ferris for excellent secretarial assistance, Adrienne Ruddock for help in preparation of the figures, $\mathrm{Dr} C$ Patterson for advice on statistical analysis, and Doctors J H Robertson and Chitra Bharucha for permission to study patients under their care.

1 Bennett JM, Catovsky D, Daniel M-T, et al. Proposals fo the classification of chronic (mature) $B$ and $T$ lymphoid the classification of chronic (mature) B and

2 El' Agnaf MR, Ennis KE, Morris TCM, et al. Successful remission induction with deoxycoformycin in elderly patients with T-helper prolymphocytic leukaemias. $\mathrm{Br} F$ Haematol 1986;63:93-104.

3 Costello C, Wardle J, Catovsky D, Lewis SM. Cell volume studies in B-cell leukaemia. $\mathrm{Br} \mathcal{f}$ Haematol 1980;45:209-14.

4 Dighiero G, Lesty CL, Couty MC, Binet JL. Valeur prognostique de l'étude des volumes lymphocytaires dans prognostique de leucémie lymphoide chronique. Nouv Rev Fr Hématol la leucemie lympho

5 Patterson KG, Goldstone AH, Richards JDM, Cawley JC Studies of cell size in lymphoproliferative disorders. $\mathrm{Br} \dot{\mathcal{F}}$ Haematol 1980;46:337-8.

6 Newton JA, Guthrie DL, Westwood, et al. Screening tests for Sézary cells in cutaneous T-cell lymphomas. Clin Lab Haematol 1986;8:109-14.

7 Davey FR, Kurec AS, Oates RP. Cell volumes of lymphoblasts in patients with acute lymphocytic leukaemia. Clin Lab Haematol 1985;7:17-26.

8 Hauser L, Kurec A, Goldberg J, et al. Prognostic significance of mean cell volume in non-Hodgkin's lymphomas. Cancer 1986;57:2363-7.

9 Melo JV, Wardle J, Chetty M, et al. The relationship between chronic lymphocytic leukaemia and prolymphocytic leuchronic lymphocytic leukaemia and prolyd

10 Melo JV, HegdeV, Parreira A, et al. Splenic B cell lymphoma with circulating villous lymphocytes: Differential diagnosis of B cell leukaemias with large spleens. F Clin Pathol 1987;40:642-51.

11 Robinson D, Lacki P, Aber V, Catovsky D. Morphometric analysis of chronic B-cell leukaemias-an aid to the classification of lymphoid cell types. Leuk Res 1989;13:357-65.

12 Orazi A, Cattoretti G, Rilke F. The Technicon H6000 analyzer discriminates chronic lymphocytic leukaemia from other B-cell leukaemias through automatic assessment of large unstained cells. Arch Pathol Lab Med 1990;114:1021-4.

13 Bessman JD, Johnson RK. Erythrocyte volume in normal and abnormal subjects. Blood 1975;46:369-79. 